\title{
Exploration and Practice on the Course Construction of "Desktop Service Comprehensive Training"
}

\author{
Yueping $\mathrm{Wu}^{1}$, Yinchun Yang $^{2}$, Ce Zhang ${ }^{3}$ \\ (12.Higher Vocational (International) College, Shanghai Polytechnic University Shanghai 201209, \\ China
}

3. Digital China, Shanghai 20033, China)

Keywords: Synthesize Training of Desktop Service; Virtualization; School-Enterprise Cooperation

\begin{abstract}
Synthesize Training of Desktop Service" is a new curriculum, according to the teaching plan, the curriculum is developed teaching content, teaching mode though enterprise cooperation, and has achieved a certain teaching effect. This innovative practice teaching mode has changed the traditional teaching mode based on the basic theory of curriculum and teaching material, and solved the limitation question of teaching system in the application, openness, prospective and interactive aspects of the knowledge system, the design of teaching content and teaching mode is worth colleges and universities learning.
\end{abstract}

\section{Introduction}

Higher vocational college in our university actively promote Shanghai modern vocational education system construction, adhere to the development road of international cooperation, the joint of production and education ,school enterprise joint, Strengthen exchange and cooperation with international vocational education, close the docking of talent training and modern industrial system, develop the skilled talents with "professional skills, professional credit, professional characteristics" in international field. According to the orientation of my college, and the important development of virtualization technology on the market today, offer a "Desktop Service Comprehensive Training "course for the computer professional students, the course closely around the market demand and enterprise development [1][2],so that students can directly go to the society for the position of desktop service engineer. According to the investigation, other higher vocational colleges have not opened desktop service course with virtualization, the concept of virtualization only in the stage of undergraduate or master[3][4],but the teaching content designed through author and the cooperation enterprise can be accepted completely by the computer professional students in the higher vocational college, and the course has been opened for two years, to achieve the desired effect.

This course mainly involves two aspects of service and desktop content. Improve the students' ability to analyze and solve problems, the ability to write documents and master effective communication skills through learning; Master installation, packaging of Windows system, manage the machine environment, the various types of configuration and management about network services; Understand the architecture, working principle about desktop virtualization based on VMWare View, configure environment, build a platform, and achieve to deploy the flexible virtual desktop architecture. Desktop virtualization is one aspect of virtualization technology, which separate the operating system from hard disk, memory, CUP and other hardware in simple terms, the operating system is not running in the actual existing physical devices, but the virtual devices[5][6]. It is a common client solution that can be used separately to manage the operating systems, hardware, applications program and users; In the management, no matter where they reside, will not interfere each other.

This course is to make full use of the cooperative mode of production and education [7], to carry out that engineers inside and outside the school cooperate to teach, learn cognitively in enterprise, and practice in school and other forms, completely solve the traditional teaching mode based on curriculum theory and teaching material and the limitation problems that are applicability, openness, 
forward-looking and interactive of knowledge system in the teaching system [8].

\section{Clear Teaching Object and Training Goal}

As higher vocational colleges, training of talents, should not only have the practical experience, but be combined with the market, higher education has a great advantage in the traditional education theory and research personnel training of computer major in general. But there is single level, lag of teaching content, a serious gap between theory and practice in the cultivation of applied talents, the curriculum is obsolete, facilities can't keep up, so now most of the graduates are more than theoretical, the relative lack of application technology required in professional positions. Therefore, it is necessary to set up the curriculum through the cooperation with the corresponding professional enterprise, we also continue to learn experience in the process of enterprise and communication, and gradually improve the curriculum which make it more adapt to the needs of the job, that make them more handy when students enter the workplace, more potential in the development path of the occupation.

\section{Teaching object.}

This course covers the virtual desktop technology and single operation services which is the most popular in the information industry, is a practical professional training course in the short term, students are required to have basic computer knowledge, LAN technology and components, can be used as a required course for students majoring in computer application technology, network, and other professional courses.

\section{The market-oriented teaching aim}

IT desktop is almost a touch thing everyone use IT facilities. These IT desktop devices provide a number of applications and services, is foundation the staff can work normally in the information age, as shown in figure 1-1. Its management has almost become the organization's daily work for user of the IT desktop, its importance is self-evident. Work supported by IT desktop on the surface, is only "enabled" equipment, but in essence, it make employees work normally, and ensure that it is integral part that business processes of a department or an organization can be operated normally . So, I t has a different general significance.

According to the exchange with the enterprise, summed up the main job requirements of the desktop operation and maintenance engineers: 1. Responsible for resolving hardware problems, including desktop troubleshooting, desktop maintenance, and technical support. 2. Responsible for resolving the emergence problem of the desktop system, including the installation and maintenance of office software, computer operating systems, printer drivers.3. Responsible for solving common mailbox, debugging and configuration of OA system. 4. Configure the removable storage device. 5. Build a domain controller and two level sub-domain controller, and deal with domain controller problems. Back up data (AD data, DNS data, system disk data) to the machine at the domain controller level and store backup data (local and remote). 6. Write scripts, achieve the configuration of the client to change through the domain controller, and scheduled task settings. 7. Functional implementation of antivirus software, enterprise planning and deployment, and hierarchical architecture. 8, the rights of various levels of windows machines are divided. 9, build and set up DNS server. 10. Configure ODBC of the SQL database, and back up the SQL database data. 11. able to provide remote network services, to understand the basic knowledge of the network.12. Summarize daily work, write a variety of pre-market technical documentation, and design documents according to customer requirements. And now the application of desktop virtualization is very mature in the enterprise, but also breed a new task is to create, configure, migrate, manage and monitor virtual machines and virtual devices, manage user access to virtual infrastructure.

"Desktop Service Comprehensive Training "course is not only for the training of desktop operation and maintenance engineers, but also to adapt to the work, learning and lifestyle in the cloud era, so that more people know, understand, master and apply virtualization. 


\section{The Teaching Mode, Content and Effect in the Mode of School - enterprise Cooperation}

Teaching method.

In addition to the normal school hours, students in our higher vocational college are required to have a

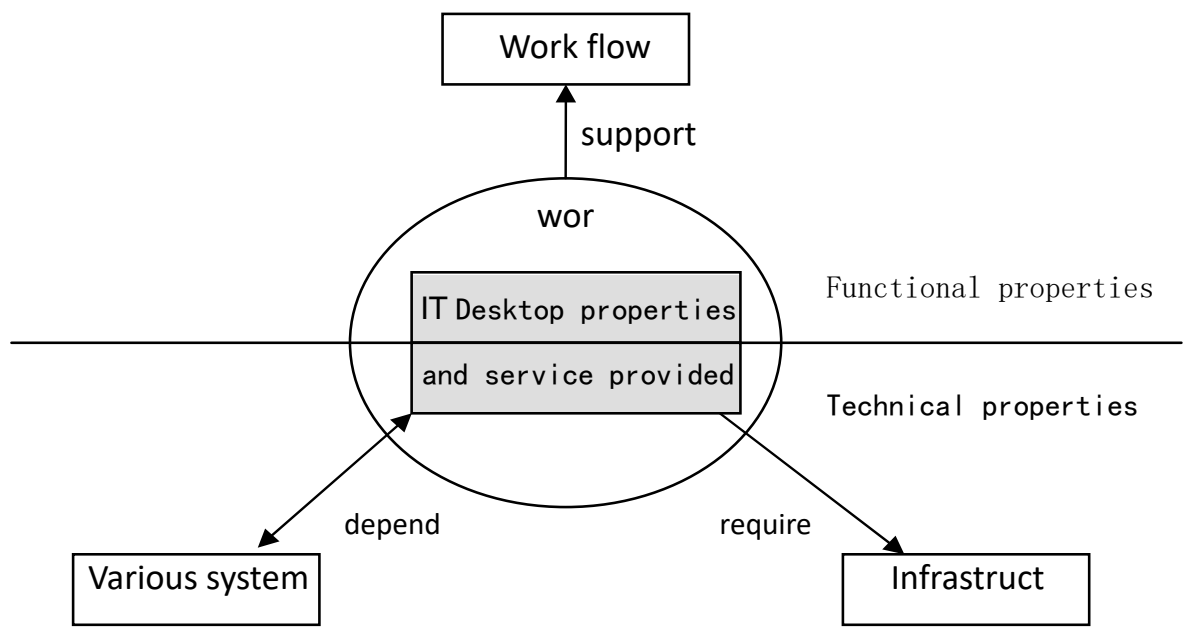

Fig.1 IT Desktop

certain time of internship and achievements in textual research before graduation, "Desktop Service Comprehensive Training "course is put in short term a year before graduation, is taught in the form of "lectures, operation, visit, summarize and exchange ", and has taught qualified enterprises engineers with" 1:2 "the proportion of cooperative teaching, 48 hours of classes, 16 hours in the enterprise by the Engineer teaching. Achieve in-depth cooperation with enterprises through this course, grant students service quality to make customer satisfaction from the perspective of the enterprise, including basic communication skills, ability of solve and analyze problems and document writing skills; Lead students to install, deploy the virtual environment from the actual experience of the enterprise, teach the principle and architecture of the virtual desktop; Students enter into the enterprise cognitive learning for another 4 hours. The overall report assess in the form of report, students reorganize the contents of the study, learning experience, the operation process, the results of the experiment through the report. The remaining 32 hours completed by school teachers, guide the student to carry on the system installation, encapsulation, the machine domain, the installation and configuration of active directory, DNS and DHCP.

\section{Course Content.}

According to the professional teaching plan, the course combined with the current market demand and development is made teaching content, mainly divided into: comprehensive service ability, Windows installation and application, Windows Server 2008 system configuration and desktop virtualization.

\section{1) Comprehensive Service Ability}

Improve the ability of analyzing and solving problem, guide students to learn to convert the thinking with a positive attitude, use scientific and accurate analysis method to define clearly the problem; Master the methods of document writing, and understand the pyramid writing principle; Achieve effective communication.

\section{2) Windows Installation and Application}

Install Windows 7 and Window Server 2008 system in different mode; Master Windows 7 system package production method; Master disk and equipment management; configure file access and network connection on the client.

\section{3) Windows Server 2008 System Configuration}

Understand the meaning of domain and active directory, set up the domain environment, use the 
domain account management, Centralized management and control of users and computers in a domain by using group policy based on Windows Server 2008. Learn configuration method of offline folder, shadow copy in server and client. Learn to create and access methods of distributed file systems. Configure DNS, DHCP, and VPN services according to application requirements. Install virtualization service Hyper-V on Server Windows 2008 server, configure Hyper-V, including basic settings, network settings. Create a virtual machine in Hyper- $\mathrm{V}$ and install the operating system. Modify the settings of the virtual machine after the installation is complete.

\section{4) Desktop Virtualization}

View VMware is based on the industry's leading virtualization platform. Its application is already mature in schools and enterprises, at present, computer and information institution, Faculty of Engineering in our school, laboratory has been trying to use the VMware to manage virtually server and desktop[9][10], make full use of the server resources, to reduce the requirements for the performance of the terminal, the computer room environment becomes more manageable. VMware View can simplify desktop and application management, reduce costs and improve data security, and provide more flexibility and IT control for end users. The use of VMware View solve the general client program, the solution can package the operating system, applications and data to the isolation layer, the IT staff can individually change, update and deploy each layer, so as to get more business flexibility. Customers can extend VMware infrastructure and its high availability, disaster recovery and business continuity, such as the value of enterprise class capabilities to the desktop through View VMware.

Must master the knowledge and skills to build and run VMware VSphere environment. Must understand how to install and configure the ESXi VMware host and VMware VCenter Server, must also learn how to use VCenter Server to manage ESXi hosts and virtual machines.

\section{Teaching effect.}

"Desktop Service Comprehensive Training “ course has been opened for two years through the mode of school-enterprise cooperation, the initial is in the form of extra-curricular training is transformed into the required courses for computer application technology professional students, students feedback that teaching content arrangement of this course is reasonable, teaching mode innovation, has a good teaching effect through after-school report and exchange. Students are willing to take the initiative to learn the knowledge that is used, and closely integrate with the market. For the enterprise, it is no longer passive acceptance of talent recommendation, but actively tap the talent, choose the right student internship or employment from the class, this is a long-term development and win-win cooperation model.

\section{Conclusion}

"Desktop Service Comprehensive Training “ course breaks through the traditional single teaching mode, content arrangements conform to the needs of the market and the development , students' acceptance degree is improved. But the curriculum in the desktop virtualization module require the higher experimental environment, need to provide five service systems, one client for testing, one management ESXI host and one virtual machine. Therefore, the computer room has 8G memory of the computer at least, otherwise it will seriously affect the experiment progress of students and the teaching effect of teachers.

\section{References}

[1] Chang Liu, Yan Feng. Desktop Virtualization and Its Application Program in Knowledge Enterprise [J].China Information Times. 2011(8):58-61.

[2]Xiaowei Lu, Jun Xiao, Guohong Wang etc. Research on the Application of Enterprise Desktop Virtualization [C]. MIS/S\&A academic exchange conference of China Shipbuilding Engineering Society in 2012. 2012(5):222-226.

[3] Li Ming-jun, Lu Ying . Investigation on Virtualization and Cloud Computing Course 
Construction at Colleges [J].Journal of Harbin University of Commerce (Natural Sciences Edition). 2013 (29):466-469

[4] Bao Ai-hua, Chen Wei-wei, Liu Peng etc. Content system construction and practice of cloud computing courses[J]. Computer Engineering and Science. 2014(36):42-45.

[5]Qin Rui, Li Yue, Qin Wei. Great changes in the information age_-Virtualization technology introduction[J]. Internet and Information. 2012(6):34-35.

[6] Xiaofeng Wang. Research and Implementation of Desktop Virtualization System and Its Application Based on XEN[D]. School of software, Tianjin University. 2014(2).

[7] Lin Wei-li, Wu Shen-jiang, Liu Qing. Teaching pattern of enterprises set up in school-enterprise cooperation[J]. Journal of Nanchang Education College. 2013(28):69-73.

[8] Xiao Li-min, Ruan Li, Zhu Ming-fa. Research and Practice on System Virtualization Teaching Method[J]. Computer Education2010(13):64-66.

[9]Yueping Wu ,Jian Zheng, Yi Du. Building of Virtual Server in Computer Laboratory [C] . T Proceedings of the International Conference on Information Engineering and Applications, IEA 2012: 373-379.

[10] Yueping Wu, Yinchun Yang. Construction of Laboratory Platform based on Cloud Computing [C] .The 5th International Workshop on Computer Science and Engineering (WCSE 2015).2015(5):472-476. 